\title{
Chest pain triage in the ED: Is CT coronary angiography the answer?
}

\author{
Raymond J. Gibbons, MD
}

The initial evaluation and triage of patients presenting to the emergency department with chest pain represents an enormous challenge. At one end of the spectrum, patients have non-cardiac diagnoses, including chest wall pain, gastroesophageal reflux, and peptic ulcer disease. At the other end of the spectrum, patients have definite acute coronary syndromes (ACS) with either ST elevation myocardial infarction or non-ST elevation myocardial infarction with positive cardiac biomarkers and ST segment changes. In between these two extremes, there are literally millions of patients who present to the emergency department each year with possible ACS. If their initial electrocardiogram and cardiac biomarkers are not diagnostic, how should they be managed? In the past, virtually all these patients were admitted to the hospital to "rule out myocardial infarction,' a conservative but expensive approach that ensured that the small percentage of patients who really had myocardial infarction were not incorrectly sent home. The first national Unstable Angina guideline, which was commissioned by the Agency for Health Care Policy Research in the early 1990s, pointed out that many of these patients were at low risk for myocardial infarction, and could be safely sent home without admission. ${ }^{1}$ However, many hospitals and physicians were uncomfortable with this judgment and developed a series of rapid evaluation protocols within the emergency department to identify patients who could be safely discharged. These protocols, which were published in a large number of observational and registry studies, were subsequently formalized as "chest pain centers" to reduce admissions and avoid unnecessary treatment. More than 550 hospitals in the United States currently

From the Division of Cardiovascular Disease, Department of Internal Medicine, Mayo Clinic, Rochester, MN.

Dr Gibbons is a consultant for Lantheus Medical Imaging.

Reprint requests: Raymond J. Gibbons, MD, Division of Cardiovascular Disease, Department of Internal Medicine, Mayo Clinic, Gonda 5, 200 First Street S.W., Rochester, MN 55905; gibbons. raymond@mayo.edu.

J Nucl Cardiol 2012;19:404-6.

1071-3581/\$34.00

Copyright (C) 2012 American Society of Nuclear Cardiology.

doi: $10.1007 / \mathrm{s} 12350-012-9567-0$ have such centers, many of which are formally accredited by the Society of Chest Pain Centers.

Randomized trials of chest pain triage are very challenging. They require coordination of hospital procedures, emergency department physicians, and cardiologists in support of a standardized protocol that permits identification of those few patients who have myocardial infarction to ensure patient safety. Two noteworthy randomized trials were conducted during the 1990s. One was a single-center study from the Mayo Clinic $^{2}$ that randomized patients with intermediate-risk unstable angina to routine admission, or evaluation in a chest pain unit within the emergency department. The chest pain unit monitored patients for 6 hours and performed serial biomarkers. Patients who had negative biomarkers and no further chest pain then underwent treadmill exercise ECG testing whenever possible. Stress imaging with echocardiography or myocardial perfusion imaging was performed in a minority of patients $(18 \%)$ in whom treadmill exercise testing was not feasible. ${ }^{3}$ Patient outcomes were similar in both randomization groups. Admissions and total costs were reduced in patients triaged to the chest pain unit.

The second randomized trial ${ }^{4}$ was a large multicenter trial of 2,475 patients that randomized patients to standard care (including chest pain units in some hospitals) with or without rest myocardial perfusion imaging in the emergency room. Rest myocardial perfusion imaging reduced admissions in those patients who proved not to have ACS, although not in one of the seven participating centers (which had a very low admission rate). These two randomized trials (and other observational studies) supported the recommendations in the 2007 ACC-AHA unstable angina-non-ST elevation myocardial infraction guidelines ${ }^{5}$ that patients with possible ACS should be observed for at least 12 hours from symptom onset and then undergo a stress study to provoke ischemia. An exercise or pharmacologic stress test to provoke ischemia is a Class I recommendation in these guidelines. If this is negative, the patient should be followed up as an outpatient. If this is positive, the patient should be admitted to the hospital and treated as acute coronary ischemia

The guidelines do recognize a potential role for CT coronary angiography in a separate Class IIa recommendation - "the performance of a non-invasive coronary imaging test (CCTA) is reasonable as an 
alternative to stress testing." Four recent randomized trials have examined the potential role of CT coronary angiography in chest pain triage. The first was a singlecenter study at William Beaumont Hospital ${ }^{6}$ that randomized 197 patients to CT coronary angiography or SPECT myocardial perfusion imaging. This trial found that major adverse cardiac events (MACE) were equivalent in the two groups. Costs in the emergency department were reduced with CT angiography. Unfortunately, total costs during the episode of care were not measured. These promising results led to three larger multi-center trials. ${ }^{7-9}$ Two of these have been published; the third ${ }^{9}$ was presented at the 2012 American College of Cardiology meeting, and will presumably be submitted for publication shortly.

In the current era of healthcare reform, what are the preferred primary endpoints of such trials? They should certainly ensure patient safety by measuring major cardiac events, not only during the hospitalization but also during the following months. Assuming equivalent patient outcomes, the primary goal of chest pain triage should be to reduce hospital admissions, both for patient satisfaction and for cost reduction. The earlier Mayo randomized trial performed a very detailed cost analysis. The treadmill exercise test was assigned a "relative cost" of 1 . Events in the emergency room, including imaging, had "relative costs" that were generally $<10$. In contrast, admission to a monitored bed in the hospital had a relative cost of 25 , coronary angiography had a relative cost of 30 , percutaneous coronary intervention (PCI) had a relative cost of 94, and coronary artery bypass grafting (CABG) had a relative cost of 205. Although, these relative cost estimates were developed in the 1990s, they are probably still valid today (although current absolute costs would certainly be higher). Admissions are, therefore, a major " cost driver." However, a reduction in admissions will not necessarily reduce total costs, since one admission with CABG will cost more than 8 admissions without CABG. For that reason, measurement of total costs is another preferred endpoint in randomized trials of chest pain triage.

What are the results of the three recent randomized trials of CT coronary angiography with respect to these preferred primary endpoints? All three trials show equivalent patient outcomes with or without CT coronary angiography. CT coronary angiography had a variable effect on hospital admissions. In the CT-STAT trial, " "hospital admission" is not specifically described. However, if "invasive angiography" is a surrogate for admission. $6.2 \%$ of the patients in the standard of care group underwent angiography and $6.6 \%$ of the patients in the $\mathrm{CT}$ coronary angiogram group underwent angiography; this difference was not statistically significant. In the trial by Litt et $\mathrm{al}^{8}{ }^{8}$ direct discharge from the emergency department was more common in the CT angiography group (50\% vs $23 \%$ ), but the remaining patients are listed as "admission or observation." It is, therefore, not clear how many of these patients were actually admitted to the hospital versus observed in a chest pain unit. The ROMICAT II trial by Hoffman et $\mathrm{al}^{9}$ is the most transparent regarding admissions. "Direct ED discharge", was more common in the CT coronary angiography group (46\% vs $12.4 \%$ ). However, "admission to an observation unit" was more common in the standard of care group (53.7\% vs $26.6 \%$ ), and "admission to the hospital" was reduced only modestly with CT angiography (25.4\% with CT angiography vs $31.7 \%$ with standard care, $P=.027)$. As described previously, "admission to the hospital" is more important from a cost standpoint.

What about total costs? Only the ROMICAT II trial actually measured these. Despite a reduction in admissions, total costs were not significantly different between the two groups $(\$ 4,004 \pm 6907$ in the CT coronary angiography group vs $\$ 3,828 \pm 5289$ in the standard care group). (The large standard deviation in both groups suggests a very skewed distribution, likely reflecting cost outliers due to revascularization.)

All three randomized trials of CT coronary angiography reported an advantage for $\mathrm{CT}$ angiography with respect to shorter time in the ED, which should reduce ED overcrowding and be desirable from a patient standpoint. If outcomes and total costs were neutral, and admissions were somewhat reduced by CT angiography, should we favor CT angiography on the basis of the shorter stay in the ED? Although these trials provide important evidence, I do not think they definitely answer this question. There is a potential "downside" to this approach that is evident from careful review of the results. In all 4 trials, the rate of coronary revascularization with PCI or CABG was higher in the CT coronary angiography group (Table 1). Although this difference did not achieve statistical significance in any individual trial, it is likely that a pooled analysis would achieve statistical significance. These excess revascularization procedures were performed in patients without ECG changes, biomarkers, or stressinduced ischemia. An evidence-based review would likely question their necessity. Only a small minority of those patients with significant anatomic disease on invasive coronary angiography underwent revascularization, reflecting a relatively conservative approach in these centers. Given the well-recognized regional variations in PCI and CABG rates in the Dartmouth Healthcare Atlas, the rate of coronary revascularization might conceivably have been higher in other health care markets. Only a single trial has measured total costs. Given the large relative expense (and non-zero risk) of coronary revascularization, these studies may well have been 
Table 1. Percentage of patients undergoing coronary revascularization in randomized trials of CT coronary angiography for ED chest pain triage

\section{References}

Beaumont
Goldstein (CT-STAT)
Litt
Hoffman (ROMICAT II)

6

7

Std. care group (\%)

CTA group (\%)

1.0

5.0

2.4

3.6

$8-1.3$

2.7

4.2

6.4

Std, Standard; CTA, CT coronary angiography.

" 'under-powered' ' to detect short-term differences in total costs and adverse event rates related to these revascularization procedures. Given their uncertain long-term benefits, the overall value of these revascularization procedures is unclear.

Given these results, what can an evidence-based physician conclude? CT coronary angiography is certainly a reasonable alternative to stress testing, as reflected in the Class IIa recommendation in the existing guidelines. In individual hospitals, where ED overcrowding and "boarding", is a major issue, clinicians may prefer this option to standard stress testing. However, ED overcrowding could also be reduced by other approaches. Better screening of patient telephone calls could potentially identify low-risk patients who can be safely evaluated as outpatients without going to the ED. DeBusk et $\mathrm{al}^{10}$ have developed a clinical decision support tool for this purpose. Another approach is the use of decision aids in the ED. The Chest Pain Choice Trial at Mayo found that patients who used a decision aid were $19 \%$ less likely to be admitted to the chest pain unit for stress testing. ${ }^{11}$

The use of CT angiography in the ED should ideally be a "shared decision" between patients and physicians. Patients should understand that the available evidence suggests that this approach has no definite advantage in overall outcomes or total costs. It will likely shorten their stay in the emergency department, and perhaps decrease their chances of hospital admission, but potentially lead to interventional procedures (PCI and $\mathrm{CABG}$ ) that may not be justified and that will certainly lead to more prolonged hospital stays. In patients with interpretable ECGs who are able to exercise, the treadmill ECG approach reported by Mayo carries no radiation exposure, has no increase in adverse outcomes at 5 years and reduces total costs. ${ }^{12}$ On the basis of current evidence, it continues to offer great value.

\section{References}

1. Braunwald E, Jones RH, Mark DB, et al. Diagnosing and managing unstable angina: Quick reference guide for clinicians, No. 10. Rockville, MD: Agency for Health Care Policy and Research, US Dept of Health and Human Services. AHCPR Publication: 940603 (1994).

2. Farkouh ME, Smars PA, Reeder GS, et al. A clinical trial of chestpain observation unit for patients with unstable angina. N Engl J Med 1998;339:1882-8.

3. Ramakrishna G, Milavetz J, Zinsmeister AR, et al. Effect of exercise treadmill testing and stress imaging on the triage of patients with chest pain: CHEER substudy. Mayo Clin Proc 2005;80:322-9.

4. Udelson JE, Beshansky JR, Ballin DS, et al. Myocardial perfusion imaging for evaluation and triage of patients with suspected acute cardiac ischemia: A randomized controlled trial. JAMA 2002;288:2693-700.

5. Anderson JL, Adams CD, Antman EM, et al. ACC/AHA 2007 guidelines for the management of patients with unstable angina/ non-ST-elevation myocardial infarction. J Am Coll Cardiol 2007;50:e1-157.

6. Goldstein JA, Gallagher MJ, O'Neill WW, Ross MA, O’Neil BJ, Raff GL. A randomized controlled trial of multi-slice coronary computed tomography for evaluation of acute chest pain. J Am Coll Cardiol 2007;49:863-71.

7. Goldstein JA, Chinnaiyan KM, Abidov A, et al. The CT-STAT (Coronary Computed Tomographic Angiography for Systematic Triage of Acute Chest Pain Patients to Treatment) trial. J Am Coll Cardiol 2011;58:1414-22.

8. Litt H, Gatsonis C, Snyder B, et al. CT Angiography for Safe Discharge of Patients with Possible Acute Coronary Syndromes. N Engl J Med 2012;366:1393-1403.

9. http://www.theheart.org/article/1376891.do. Accessed April 3.

10. DeBusk RF, Miller NH, Raby L. Technical feasibility of an online decision support system for acute coronary syndromes. Circ Cardiovasc Qual Outcomes 2012;3:694-700.

11. Hess EP, Knoedler MA, Shah ND, et al. The chest pain choice decision aid. Circ Cardiovasc Qual Outcomes 2012;5. doi: 10.1161/CIRCOUTCOMES.111.964791.

12. Cullen MW, Reeder GS, Farkouh ME, et al. Outcomes in patients with chest pain evaluated in a chest pain unit: The chest pain evaluation in the emergency room study cohort. Am Heart $\mathbf{J}$ 2011;161:871-7. 